\title{
Panus lecomtei (Fr.) Corner (Agaricomycetes, Polyporales, Panaceae): primeira ocorrência no Estado do Piaú, Brasil
}

\author{
Maria das Graças Miranda Nunes' ${ }^{1}$, (1) Mateus Oliveira da Cruz $^{2}$ e (1) Maria Helena Alves ${ }^{1,3}$
}

Submetido: 19.02.2020; aceito: 18.08.2020

Como citar: Nunes, M.G.M., Cruz, M.O. \& Alves, M.H. 2020. Panus lecomtei (Fr.) Corner (Agaricomycetes, Polyporales, Panaceae): primeira ocorrência no Estado do Piauí, Brasil. Hoehnea 47: e132020. http://dx.doi.org/10.1590/2236-8906-13/2020.

\begin{abstract}
Panus lecomtei (Fr.) Corner (Agaricomycetes, Polyporales, Panaceae): first occurrence in Piauí State, Brazil). This work was aims to study and publicize Panus lecomtei as the first occurrence for Piauí State. The collections were performed in the rural areas of Joaquim Pires and Murici dos Portelas municipalities, Cipoal and Jenipapeiro communities, respectively. The expeditions took place from 2015 to 2017. The material was photographed, collected and taken to the Botany Laboratory of the Universidade Federal do Piauí (Campus Ministro Reis Velloso), where the identification by classical method was carried out studying the macroscopic characteristics with the aid of a stereoscopic microscope and microscopes under an optical. To store the material, it was placed in an kiln for drying. The specimen has a solitary, aggregate habit, found in decaying trunks, with a leathery aspect, pubescents pileus and stipe. Lamellated hymenophore. Microscopic analysis showed basidia with 4 sterigmas; ellipsoid to ovoid basidiospores; generative and skeletal hyphae; very evident connection clamp, present cheilocystidia and clavate metuloids. The data from this research contribute to the divulgation of Panus lecomtei as first occurrence in the Piauí State and for the mycological knowledge in the Northeast region of Brazil.

Keywords: Basidiomycetes, biodiversity, northeast of Brazil, Panaceae, Polyporales
\end{abstract}

RESUMO - (Panus lecomtei (Fr.) Corner (Agaricomycetes, Polyporales, Panaceae): primeira ocorrência no Estado do Piauí, Brasil). Este trabalho teve por objetivo estudar e divulgar Panus lecomtei (Fr.) Corner como primeira ocorrência para o Estado do Piaú. As coletas foram realizadas nas zonas rurais dos municípios de Joaquim Pires e Murici dos Portelas, comunidades Cipoal e Jenipapeiro, respectivamente. As expedições ocorreram no período de 2015 a 2017. O material foi fotografado, coletado e levado para o laboratório de Botânica da Universidade Federal do Piauí (Campus Ministro Reis Velloso), onde realizou-se a identificação pelo método clássico estudando as características macroscópicas com auxílio de microscópio estereoscópio e microscópicas sob microscópio óptico. Para armazenamento do material, o mesmo foi colocado em estufa para secagem. O espécime possui hábito solitário, agregado, encontrado em troncos em decomposição, com aspecto coriáceo, píleo e estipe pubescente. Himenóforo lamelado. As análises microscópicas demonstraram basídios com 4 esterigmas; basidiósporos elipsoides a ovoides; hifas generativas e esqueléticas; grampos de conexão evidentes e presença de queilocistídios e metulóides clavados. Os dados desta pesquisa vêm contribuir para a divulgação de Panus lecomtei como primeira ocorrência no Estado do Piaú e para o conhecimento micológico da região Nordeste do Brasil.

Palavras-chave: Basidiomycetes, biodiversidade, nordeste do Brasil, Panaceae, Polyporales

\section{Introdução}

A família Panaceae Miettinem, Justo \& Hibbett, ordem Polyporales, foi descrita por Justo et al. (2017) e agrupa táxons com características específicas, tais como basidioma pileado-estipitado, sistema hifal dimítico com grampos de conexão presente, esporos hialinos, lisos com parede fina e inamiloides. Comporta os gêneros Cymatoderma Jungh. e Panus Fr., sendo este último o gênero tipo.

1. Universidade Federal do Delta do Parnaíba, Departamento de Biologia, Avenida São Sebastião, 2819, Nossa Senhora de Fátima, 64202-020 Parnaíba, PI, Brasil

2. Universidade Federal de Pernambuco, Departamento de Micologia, Avenida Professor Nelson Chaves, s/n, Cidade Universitária, 50670-420 Recife, PE, Brasil

3. Autor para correspondência: malves@ufpi.edu.br 
Panus reúne um grupo de organismos monofiléticos proposto por Corner (1981) como um gênero, entretanto possui classificação bastante discutida (Grand, 2004). Pegler (1983), estabelece como subgênero de Lentinus com base em características morfológicas, no entanto baseado em dados moleculares, Hibbett \& Vilgalys (1993) rejeitaram a classificação reestabelecendo Panus como gênero. O número de espécies para Panus, apresentado por Kirk et al. (2008), são de 25 espécies, entre as quais, oito são reportadas para o Brasil (Gugliotta et al. 2015, Maia et al. 2015, Vargas-Isla et al. 2015).

Entre os fungos Polyporales, o gênero Panus, atualmente acomodado em Panaceae, possui origem independente no hábito agaricoide (Justo et al. 2017) bem como Lentinus e Neofavolus da família Polyporaceae, como ressaltado por Seelan et al. (2015).

Agaricus strigosus Schwein. (1822) recebeu o nome de Lentinus lecomtei Fr. por Fries (1825). Por vez, L. lecomtei foi acomodado por Corner (1981) no gênero Panus ficando como Panus lecomtei (Fr.) Corner. Drechsler-Santos et al. (2012) ao estudarem os fungos lentinoides do Semi-árido do Brasil, desconsideraram a nomenclatura de Corner (1981) e atribuíram Panus neostrigosus Drechsler-Santos \& Wartchow como novo nome para Lentinus strigosus baseado em Agaricus strigosus. Todavia, dentro do gênero Panus, Panus lecomtei tem prioridade sobre Panus neostrigosus por ser o nome mais antigo (Vargas-Isla et al. 2015). Com isso, neste estudo considera-se Panus lecomtei. Muitos trabalhos como os de Sales-Campos \& Andrade (2010), Sales-Campos $\&$ Andrade (2011), Andrade et al. (2013) e Sales-Campos et al. (2013), realizados com a denominação de Lentinus strigosus, são relacionados ao cultivo em diferentes resíduos e sobre substâncias encontradas neste fungo com alto potencial no tratamento de doenças negligenciadas. No Brasil, Panus lecomtei tem sido reportado em oito Estados: Amazonas, Alagoas, Bahia, Pernambuco, Mato Grosso do Sul, Rio Grande do Sul, Santa Catarina e Paraná (Drechsler-Santos et al. 2012, Gugliotta et al. 2015, Vargas-Isla et al. 2015).

$\mathrm{O}$ estudo de fungos basidiomicetes no Estado do Piauí é ainda incipiente, onde poucos estudos como de Alves et al. (2019) e Alves et al. (2012) podem ser referidos. Com isto, a diversidade deste grupo de fungos no Estado do Piauí é pouco conhecida. Atualmente, a plataforma Flora do Brasil (2020) menciona 30 espécies de basidiomicetos para o Estado do Piauí. Gugliotta et al. (2015), levando em consideração a existência de vouchers, listam 11 espécies de Polyporales, não havendo registros de táxons da família Panaceae para o Estado. Assim, o presente estudo vem divulgar a primeira ocorrência de Panus lecomtei no Estado do Piauí, Nordeste do Brasil.

\section{Material e métodos}

Área de estudo-Aárea estudada está situada entre as zonas rurais dos municípios de Joaquim Pires ( $\left.3^{\circ} 31^{\prime} 80^{\prime \prime} \mathrm{S}, 41^{\circ} 59^{\prime} 62^{\prime \prime} \mathrm{O}\right)$ e Murici dos Portelas ( $\left.3^{\circ} 29^{\prime} 80^{\prime \prime} \mathrm{S}, 41^{\circ} 59^{\prime} 60^{\prime \prime} \mathrm{O}\right)$ sendo as localidades, as comunidades de Cipoal e Jenipapeiro (figura 1). Os dois municípios limitam com o município de Caxingó e estão localizados a menos de $300 \mathrm{~km}$ da capital Teresina (Aguiar, 2004). O tipo de vegetação predominante na região de Murici dos Portelas e Joaquim Pires é de campo cerrado, caatinga arbustiva e arbórea, floresta secundária mista, floresta decidual secundária mista e, em menor proporção, vegetação de parque. Com temperatura média entre $25^{\circ} \mathrm{Ca}$ $35^{\circ} \mathrm{C}$, clima tropical subúmido, úmido e duração do período seco de seis meses (Cepro, 2013).

Coleta - Foi realizada uma expedição para reconhecimento da área em janeiro de 2015 e nos anos seguintes foram efetuadas três expedições para coletas entre os meses de dezembro de 2016 e abril de 2017, em período chuvoso, pois é quando ocorre maior aparecimento de basidiomas. As coletas foram realizadas em lugares distintos dentro das duas comunidades e os espécimes encontrados coletados com a utilização, em alguns momentos, de estilete para a retirada dos mesmos. Também foram fotografados no substrato, e posteriormente fora do mesmo, sempre enfatizando os basidiomas em diferentes estágios de desenvolvimento.

Análise macro e microscópica - Após a coleta do material micológico, foram realizadas observações macroscópicas dos basidiomas, como medições do píleo e estipe, coloração, aspecto do píleo, substrato, sendo anotadas em uma ficha de coleta. As cores foram analisadas seguindo a carta de cores de Kornerup \& Wanscher (1978) e o material coletado foi acondicionado em envelopes feitos de jornal.

Após a análise macroscópica, o material foi transportado para o Laboratório de Botânica da Universidade Federal do Piauí, Campus Ministro Reis Velloso, posteriormente secado em estufa com temperatura de $40{ }^{\circ} \mathrm{C}$ por 24 horas. A coleta e identificação do espécime foi de acordo com a metodologia de Largent (1986), usando referências taxonômicas especializadas e chaves de identificação.

Seguindo a metodologia para a análise microscópica o material foi hidratado com hidróxido de potássio $(\mathrm{KOH})$ a $3 \%$, em seguida realizados cortes histológicos para observação das microestruturas em diferentes regiões do basidioma. Foram realizadas as análises e medições das microestruturas como basídios e basidiósporos e para melhor observação usou-se o corante de Floxina (1\%). No estudo dos basidiósporos usouse a estatística apontada por Largent \& Abell-davis (2011) que apresenta médias aritméticas $(\mathrm{Xm})$ de comprimentos $\mathrm{e}$ larguras de basidiósporos \pm desvio padrão medido para $n$ objetos; quociente do comprimento do basidiósporo pela largura (E) indicado como uma variação da faixa em n basidiósporos medidos; média dos valores $\mathrm{E}(\mathrm{Q}) \pm$ desvios padrão, além do tamanho da amostra $(\mathrm{n})=$ número total de basidiósporos medidos ( $\mathrm{x}$ ) dividido pelo número de basidiomas estudados (y), como trata a fórmula $\mathrm{n}=\mathrm{x} / \mathrm{y}$.

Após as análises, o material foi depositado no herbário HDELTA, Campus Ministro Reis Velloso da Universidade Federal do Piauí - UFPI, sob o número 5213. 


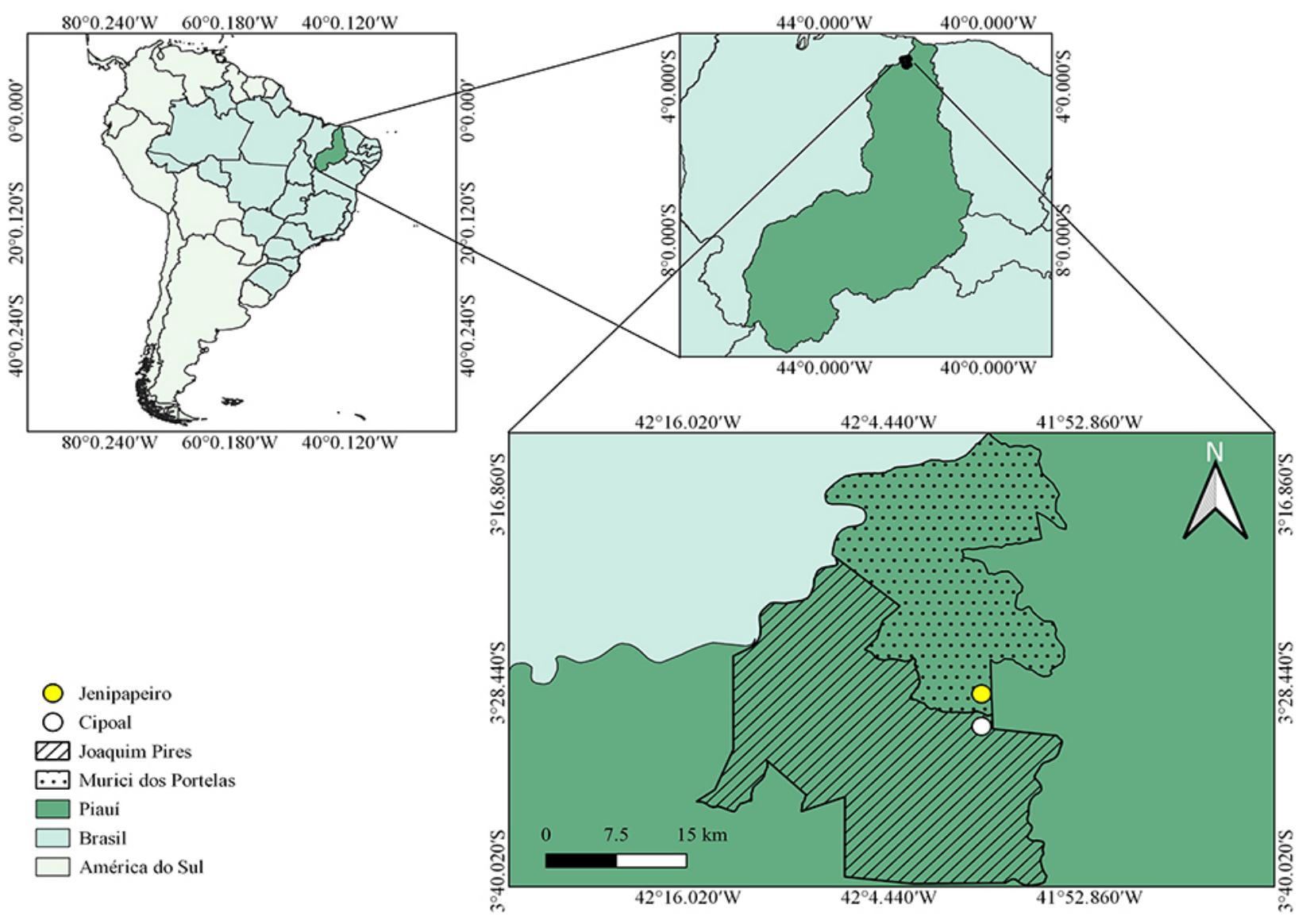

Figura 1. Mapa de localização das comunidades Cipoal (Joaquim Pires) e Jenipapeiro (Murici dos Portelas), Estado do Piauí, Brasil.

Figure 1. Localization map of Cipoal (Joaquim Pires) and Jenipapeiro (Murici dos Portelas) communities, Piauí State, Brazil.

\section{Resultados}

Os espécimes foram encontrados em troncos de árvores em decomposição, que serviam como cerca para demarcar terrenos ou sustentar casa de taipa, enquanto outros os foram em fragmentos de troncos de árvores sobre o solo em diferentes estágios do ciclo de vida, de jovem a velhos e secos.

Panus lecomtei (Fr.) Corner, Beihefte zur Nova Hedwigia 69:90 (1981).

Figuras 2 e 3

Píleo $1-5 \mathrm{~cm}$ diâm., possui aspecto coriáceo, pubescente com pequenos pelos em toda a sua superfície, umbilicado podendo ser mais profundo ou não, alguns espécimes possuem o píleo infundibiliforme, assemelhando-se a um funil, com uma variação de cores, do violáceo, inicialmente nos mais jovens, variando para tons de vinho, e tonalidades de vermelho opaco e marrom acinzentado (10D3; 10C3). Estipe 0,5-4,0 cm diâm., com aspecto coriáceo e fino, central a excêntrico, oco. A grande maioria dos espécimes possuem estipe curto, porém, eventualmente podem ser encontrados em tamanhos maiores, com coloração variando em tons violáceos à marrom, às vezes, concolor ao píleo ou diferente, pubescente com pelos menores que os encontrados no píleo. Himenóforo com lamélulas de aproximadamente quatro tamanhos diferentes, decurrentes, com tons de vermelho acastanhado e marrom acinzentado (13D4; 10C3) quando secas, ocasionalmente concolor ao píleo e estipe.

A borda da lamela é estéril, com um grande número de queilocistídios medindo 15,9-23,4 × 5,3-7,6 $\mu \mathrm{m}$, clavados, hialinos, de parede bastante fina. Pleurocistídios clavados, 24,5-34,8 $\times 6,7-8 \mu \mathrm{m}$. Possui Metuloides, 52,5-63 × 12$16 \mu \mathrm{m}$, clavados, cilíndricos, hialinos, parede espessa, ocasionalmente presentes na superfície da lamela. Trama do himenóforo irregular, hialino. Basídios 16,7-25,3 ×4,4$6,5 \mu \mathrm{m}$, cilíndricos, clavados, com quatro esterigmas. Basidiósporos 6,3-8,7 $\times 3,4-4,8 \mu \mathrm{m}(\mathrm{Xm}=7,2 \pm 0,53 \times$ $4,0 \pm 0,43 ; \mathrm{E}=1,34-2,29 ; \mathrm{Q}=1,80 \pm 0,24 ; \mathrm{n}=30 / 2)$, possuem formatos que vão de elipsoides a subgloboso, parede lisa, hialino. Possui um grande número de grampos de conexão conspícuos. Hifas generativas e esqueléticas 3,0-3,5 $\mu \mathrm{m}$ de diâmetro. As hifas generativas possuem septos e parede delgada, enquanto as hifas esqueléticas são asseptadas com parede bem evidente, devido a demarcação, ambas hialinas.

Material examinado: BRASIL. PIAuí: Joaquim Pires, Cipoal (13-I-2015) Nunes, M. G. M. (01), (HDELTA 5213). 


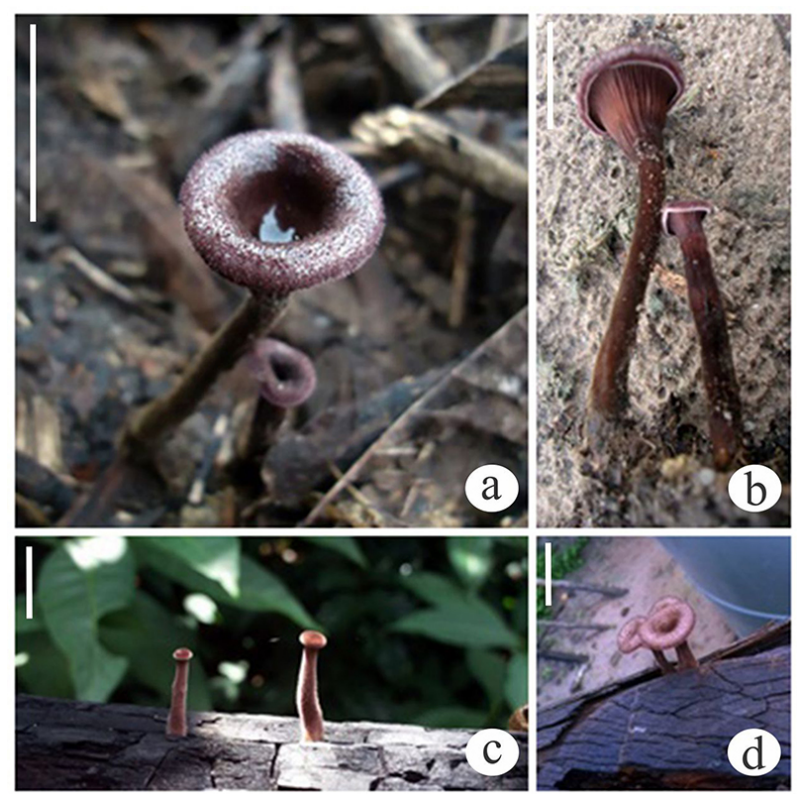

Figura 2. Panus lecomtei (Fr.) Corner. a. visão superficial do píleo. b. visão lateral do estipe. c e d. basidiomas no habitat. Fotos: Maria das Graças Miranda Nunes.

Figure 2. Panus lecomtei (Fr.) Corner. a. superficial view of the pileus. b. side view of the stipe. $\mathrm{c}$ and $\mathrm{d}$. habitat basidiomas. Photos: Maria das Graças Miranda Nunes.

\section{Discussão}

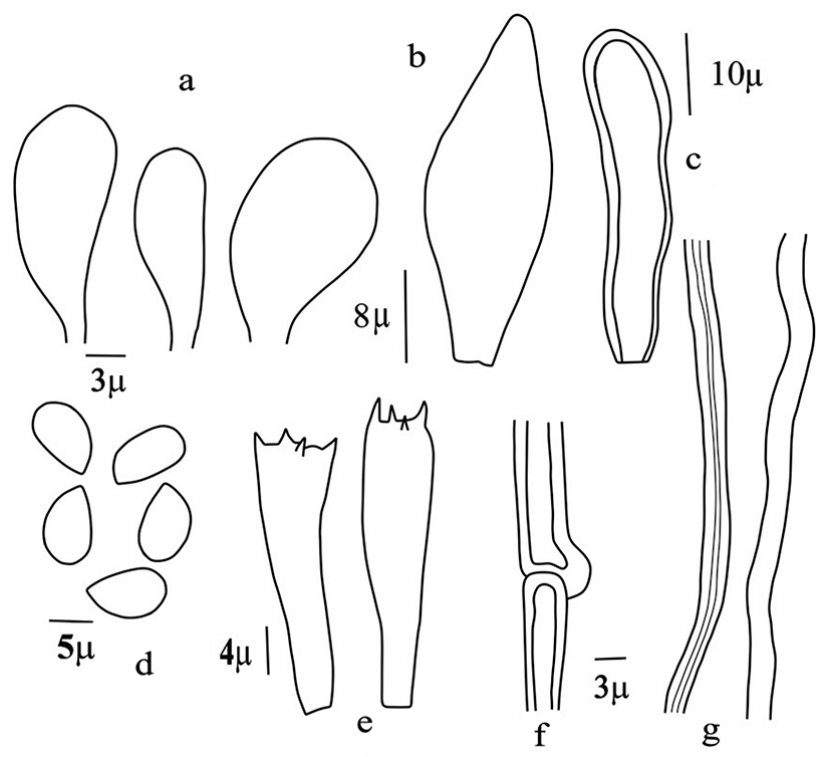

Figura 3. Panus lecomtei (Fr.) Corner. a. queilocistídios. b. pleurocistídio. c. metulóide. d. basidiósporos. e. basídio. f. hifa com grampo de conexão. g. hifa generativa e esquelética. Desenhos: Mateus Oliveira da Cruz.

Figure 3. Panus lecomtei (Fr.) Corner. a. cheilocystidia. b. pleurocystidia. c. metuloid. d. basidiospores. e. basidia. f. hiphae with connection clamp. g. generative hiphae and skeletal. Drawings: Mateus Oliveira da Cruz.
As características macroscópicas do material aqui examinado apresentam similaridade às apresentadas por Pegler (1983), com diferenças quanto ao tamanho de estruturas microscópicas como queilocistídios e basídios. Segundo o autor citado, os metuloides desta espécie podem ser abundantes ou ocasionais, corroborando com este estudo no que diz respeito aos ocasionais presentes nos exemplares examinados. Panus lecomtei HDELTA 5213 apresentou queilocistídios de tamanho menor que aqueles descritos por Vargas-Isla et al. (2015) que mediram $(12,5-)$ 22-44 (-59) $\times$ 7,5-15 $\mu \mathrm{m}$ e basidiósporos maiores comparando com os descritos pelos mesmos autores, que conferiram basidiósporos medindo 3,75-6,25 × 2,5-3,75 $\mu \mathrm{m}$. Ainda ao que se refere ao tamanho dos basidiósporos, o material aqui descrito apresentou $\mathrm{Q}=1,8 \pm 0,24$, maior que o citado por Pegler $(1983)(\mathrm{Q}=1,65)$ e Vargas-Isla et al. (2015) $(\mathrm{Q}=1,5-2)$.

Para o Brasil, além de Panus lecomtei, Maia et al. (2015) relatam: P. conchatus (Bull.) Fr., P. parvus Drechsler-Santos \& Wartchow, P. tephroleucus (Mont.) T.W. \& A. E. Wood e $P$. velutinus (Fr.) Fr. Além do mais, outras espécies de Panus tem ocorrência no Brasil, como: P. ciliatus (Lév.) T. W. May \& A.E. Wood (Putzke 1994), P. similis (Berk. $\&$ Broome) T.W. May \& A. E. Wood (Teixeira, 1946), $P$. strigellus s. 1. (Berk.) Overh. (Vargas-Isla et al. 2015) e $P$. tephroleucus (Mont.) T.W. May \& A. E. Wood (Gugliotta et al. 2015). A espécie mais semelhante à Panus lecomtei é $P$. strigellus. Estas espécies se diferenciam pela manutenção da sua cor purpúrea mesmo quando madura por P. lecomtei, além da ausência de gloeocistídio e pela presença de píleo glabrescente em $P$. strigellus.

\section{Agradecimentos}

À Universidade Federal do Piauí, Campus Ministro Reis Velloso, pela estrutura disponibilizada para a realização deste trabalho. O segundo autor agradece ao $\mathrm{CNPq}$, pela concessão da Bolsa de Iniciação Científica (PI 4345-2018) no decorrer de sua graduação.

\section{Literatura citada}

Alves, M.H., Cruz, M.O., Nascimento, C.C. 2019. First record of Chlorophyllum molybdites (G. Mey.) Massee (Basidiomycota, Agaricaceae) from Piauí state, Brazil. Check List 15: 695- 699.

Alves, M.H., Nascimento, C.C., Andrades, E.O., Araújo, L.R.C. 2012. Fungos da APA Delta do Parnaíba, litoral piauiense. In: A. Guzzi (ed.). Biodiversidade do Delta do Parnaíba: litoral piauiense. EDUFPI, Parnaíba, pp. 34-61.

Aguiar, R.B. 2004. Projeto cadastro de fontes de abastecimento por água subterrânea, Estado do Piauí: Diagnóstico do município de Joaquim. Disponível em http://rigeo.cprm.gov.br (acesso em 20-IX-19). 
Andrade, M.C.N., Sales-Campos, C., Carvalho C.S.M., Aguiar, L.B. \& Minhoni, M.T.A. 2013.Uso de resíduos madeireiros da Amazônia brasileira no cultivo in vitro de Lentinus strigosus. Ambiência 9: 189-196.

Cepro (Fundação Centro de Pesquisas Econômicas e Sociais do Piauí). 2013. Diagnóstico Socioeconômico: Joaquim Pires. Governo do Piauí. Disponível em http:// www.cepro.pi.gov.br (acesso em 20-VI-2019).

Corner, E.J.H. 1981. The agaric genera Lentinus, Panus and Pleurotus with particular reference to Malaysian species. Nova Hedwigia 69: 1-169.

Drechsler-Santos, E.R., Wartchow, F., Coimbra, V.R.M., Gibertoni, T.B. \& Cavalcanti, M.A.Q. 2012. Studies on lentinoid fungi (Lentinus and Panus) from the semi-arid region of Brazil. The Journal of the Torrey Botanical Society 139: 437-446.

Flora do Brasil em construção. 2020. Jardim Botânico do Rio de Janeiro. Disponível em http://floradobrasil.jbrj. gov.br (acesso em 23-IV-2020).

Fries, E.M. 1825. Systema orbis vegetabilis. Parte I. Lundae.

Grand, E.A. 2004. Systematics and species concepts in the genera Lentinus Fr. and Panus Fr., with enphasis on the Lentinus tigrinus, L. crinitus and Panus lecomtei complexes. Tese de Doutorado, University of Tennessee, Knoxville.

Gugliotta, A. M., Gibertoni, T. B., Drechsler-Santos, E.R., Silveira, R.M.B., Chikowski, R.S. Pires, R.M., Montoya, C.A.S., Souza, J.F., Palacio, M. \& Rezende, D.H.C. 2015. Polyporales in Lista de Espécies da Flora do Brasil. Disponível em http www.floradobrasil.jbrj. gov.br (acesso em 18-XI-2019).

Hibbett, D.S. \& Vilgalys, R. 1993. Phylogenetic relationships of Lentinus (Basidiomycotina) inferred from molecular and morphological characters. John Wiley \& Sons, New York. Systematic Botany 18: 409433.

Justo, A., Miettinen, O., Floudas, D., Ortiz-Santana, B., Sjokvist, E., Lindner, D., Nakasone, K., Niemela, T., Larsson, K.H., Ryvarden, L., Hibbett, D.S. 2017. A revised family- level classification of the Polyporales (Basidiomycota). Fungal Biology 121: 798-824.

Kirk, P.M., Cannon, P.F., Minter, D.W., Stalpers, J.A. 2008. Ainsworth \& Bisby's Dictionary of the Fungi, 10 ed. CABI, Wallingford.

Kornerup, A. \& Wanscher, J.H. 1978. Methuen Handbook of Colour, 3 ed. Eyre Methuen, London.

Largent, D.L. \& Abell-Davis, S.E. 2011. Observations on Inocephalus virescens comb. nov. and Alboleptonia stylophora from northeastern Queensland. Mycotaxon 116: 231-245.
Largent, D.L. 1986. How to Identify Mushrooms to Genus I: Macroscopic features. I. 2 ed. Mad River Press, California.

Maia, L.C., Carvalho Jr, A.A., Cavalcanti, L.D.H., Gugliotta, A.M., Drechsler-Santos, E.R., Santiago, A.L.M.A., Cáceres, M.E.S., Gibertoni, T.B., Aptroot, A., Giachini, A.J., Soares, A.M.S., Gomes-Silva, A.C., Magnago, A.C., Goto, B.T., Lira, C.R.S., SalvadorMontoya, C.A., Pires-Zottarelli, C.L.A., Silva, D.K.A., Soares, D.J., Rezende, D.H.C., Luz, E.D.M.N., Gumboski, E.L., Wartchow, F., Karstedt, F., Freire, F.M., Coutinho, F.P., Melo, G.S.N., Sotão, H.M.P., Baseia, I.G., Pereira, J., Oliveira, J.J.S., Souza, J.F., Bezerra, J.L., Araujo Neta, L.S., Pfenning, L.H., Gusmão, L.F.P., Neves, M.A., Capelari, M., Jaeger, M.C.W., Pulgarín, M.P., Menolli Jr, N., Medeiros, P.S., Friedrich, R.C.S., Chikowski, R.S., Pires, R.M., Melo, R.F., Silveira, R.M.B., Urrea-Valencia, S., Cortez, V.G. \& Silva, V.F. 2015. Diversity of brazilian fungi. Rodriguésia 66: 1033-1045.

Pegler, D.N. 1983. Agaric Flora of the Lesser Antilles. 1 ed. Kew Bulletin Additional Series IX, London.

Putzke, J. 1994. Lista dos fungos Agaricales (Hymenomycetes, Basidiomycotina) referidos para o Brasil. Caderno Pesquisa Série Botânica 6: 1-189.

Sales-Campos, C. \& Andrade, M.C.N. 2010. Temperatura e Meio de cultura mais favoráveis ao crescimento micelial de uma linhagem de Lentinus strigosus de ocorrência na Amazônia. Arquivos do Instituto Biológico 77: 539-543.

Sales-Campos, C. \& Andrade, M.C.N. 2011. Aproveitamento de resíduos madeiros para o cultivo do cogumelo comestível Lentinus strigosus de ocorrência na Amazônia. Acta Amazonica 41: 1-8.

Sales-Campos, C., Araújo, L.M., Minhoni, M.T.A. \& Andrade, M.C.N. 2013. Centesimal composition and physical-chemistry analysis of the edible mushroom Lentinus strigosus occurring in the Brazilian Amazon. Anais da Academia Brasileira de Ciências 85: 1537-1544.

Seelan, J.S., Justo, A., Nagy, L.G., Grand, E.A., Redhead, S.A. \& Hibbett, D. 2015. Phylogenetic relationships and morphological evolution in Lentinus, Polyporellus and Neofavulus, emphasizing South-eastern Asian taxa. Mycologia 107: 460-474.

Teixeira, A.R. 1946. Himenomicetos brasileiros - III. Bragantia 6: 165-188.

Vargas-Isla, R., Capelari, M., Meloni, N., Nagasawa, E., Tokimoto, K. \& Ishikawa, N.K. 2015. Relationship between Panus lecomtei and P. strigellus inferred from their morphological, molecular and biological characteristics. Mycoscience. 56: 561-571. 